MPP-2008-75

$\mathrm{MZ}-\mathrm{TH} / 08-21$

$\mathrm{SFB} / \mathrm{CPP}-08-45$

\title{
Top-quark pair + 1-jet production at next-to-leading order QCD
}

\author{
S. Dittmaier ${ }^{\mathrm{a} *}$, P. Uwer ${ }^{\mathrm{b} \dagger}$, S. Weinzierl ${ }^{\mathrm{c}}$ \\ ${ }^{a}$ Max-Planck-Institut für Physik (Werner-Heisenberg-Institut), D-80805 München, Germany \\ bInstitut für Theoretische Teilchenphysik, Universität Karlsruhe, D-76128 Karlsruhe, Germany \\ ${ }^{\mathrm{c} U n i v e r s i t a ̈ t ~ M a i n z, ~ D-55099 ~ M a i n z, ~ G e r m a n y ~}$
}

Top-quark pair production with an additional jet is an important signal and background process at the LHC. We present the next-to-leading order QCD calculation for this process and show results for integrated as well as differential cross sections.

\section{Introduction}

With a mass of $172.6 \mathrm{GeV}$ the top-quark is by far the heaviest elementary fermion in the Standard Model (SM). Its mass is more than 30 times larger than the mass of the next heaviest fermion, the bottom-quark. The large mass has lead to various speculations whether the top-quark behaves as a normal quark or whether it plays a special role. In particular, the fact that the top-quark mass is close to the scale of electroweak symmety breaking-or equivalently, that the Yukawa coupling to the Higgs is very close to one-has motivated different scenarios in which the top-quark drives the electroweak symmetry breaking. For a recent overview we refer the interested reader to Refs. [12]. In the context of the SM the top-quark interactions are completely determined through the gauge structure. The only free parameter appearing in top-quark physics is the top-quark mass. Once this parameter is measured all remaining properties are predicted. An important task for the ongoing Tevatron collider and the upcoming LHC is the precise measurement of the top-quark properties. In this context the production of a top-quark pair togehter with an additional jet is an important reaction. This becomes already clear from the simple observation that a substantial number of events in the

\footnotetext{
${ }^{*}$ Supported in part by the European Community's Marie-Curie Research Training Network HEPTOOLS under contract MRTN-CT2006-035505

${ }^{\dagger}$ Supported as Heisenberg Fellow of the Deutsche Forschungsgemeinschaft DFG and by the DFG Sonderforschungsbereich/Transregio 9 "Computergestützte Theoretische Teilchenphysik" SFB/TR9.
}

inclusive top-quark sample is accompanied by an additional jet. Depending on the energy of the additional jet the fraction of events with an additional jet can easily be of the order of $10-30 \%$. For a more precise understanding of the topology of top-quark events it is thus important to have also an improved understanding of top-quark pair production together with a jet. Recently it has been argued that the inclusive topquark pair cross section can be measured at the LHC with an experimental uncertainty of about $5 \%$. The available theoretical predictions at next-to-leading order (NLO) togehter with resummation lead to a theoretical uncertainty of more than $10 \%[3|4| 5]$. The dominant uncertainy comes from the residual scale dependence, while the uncertainties due to the parton distribution functions are comparably small. In Ref. [3] parts of the full next-to-next-to-leading order (NNLO) were derived from general arguments. It has been shown that an NNLO calculation will reduce the theoretical uncertainty down to 5\%. Apart from the two-loop corrections (where progress has been made recently [67]) one important ingredient in going to $\mathrm{NNLO}$ are the one-loop corrections to $\overline{\mathrm{t}}+1$-jet production. Apart from its significance as signal process it turns out that $\mathrm{t} \overline{\mathrm{t}}+1$-jet production is also an important background to various new physics searches. A prominent example is Higgs production via vectorboson fusion. This reaction represents an important discovery channel for a SM Higgs boson in the mass range of 120-180 GeV. The major background to this reaction is due to $\mathrm{t} \overline{\mathrm{t}}+1$-jet [8], again underlining the need for precise theoretical predictions for this process. It is well known that predictions at leading-order 
(LO) in the coupling constant of QCD are plagued by large uncertainties. In many cases the LO predictions in QCD give only a rough estimate. Only by including NLO corrections a quantitative reliable prediction can be obtained. Given that the conceptual problems of doing such calculations are solved since quite some time, one might think that doing the required calculations should be a straightforward task. Unfortunately it turns out that this is not the case. The calculation of QCD corrections for $2 \rightarrow 3$ and $2 \rightarrow 4$ reactions is still a highly non-trivial task-not speaking about reactions with an even higher multiplicity. In general the problem can be attributed to the fact that the corrsponding matrix elements are complex functions of many variables so that an analytic treatment is no longer feasible due to the large size of the expressions. A solution to this problem is to resort to numerical methods. While in principle fine one is in many cases plagued by numerical instabilities and the long runtime. In particular, the reduction of one-loop tensor integrals to scalar one-loop integrals is in general difficult to do in a numerically stable way. In that context the calculation of the one-loop corrections to top-quark pair production with an additional jet is also interesting as a benchmark process for the developement of new methods. In the present article we will briefly comment on the calculation of the NLO corrections. In addition we will show results for integrated quantities as well as for differential distributions.

\section{Calculation}

\subsection{Born approximation}

In Born approximation the partonic reactions are $g g \rightarrow \mathrm{t} \overline{\mathrm{t}} g, q \bar{q} \rightarrow \mathrm{t} \overline{\mathrm{t}} g, q g \rightarrow \mathrm{t} \overline{\mathrm{t}} q$, and $g \bar{q} \rightarrow \mathrm{tt} \bar{q}$. The last three reactions are related by crossing. In Born approximation various well-tested approaches to calculate the required matrix elements exist. We used recurrence relation à la Berends and Giele [9] and a Feynman-diagram-based approach. In both cases four-dimensional helicity methods were employed. In addition we also used Madgraph [10] for checking. We found complete agreement of the different methods. The explicit LO calculation shows indeed the large scale dependence as expected. Without going into details we just mention that in $\mathrm{LO}$ the importance of the individual partonic channels does not follow the pattern known from inclusive top-quark pair production. While at the Tevatron the situation $t \bar{t}+1$-jet production is similar to the inclusive reaction, that is the total cross section is dominated by the quark-antiquark channel followed by the gluon-fusion process, the situation at the LHC is different from inclusive production. The most important channel is gluon fusion, but in contrast to inclusive production the second important channel is the $q g$-channel. This is due to the large parton luminosity for this channel and due to a sizeable partonic cross section.

\subsection{Virtual corrections}

The virtual corrections consist of the one-loop corrections to the LO reactions. One can classify the corrections into self-energy corrections, vertex corrections, box-type corrections, and pentagon-type corrections where all the external legs are connected to one loop thus forming a pentagon. The latter are the most complicated ones owing to their complexity and the involved tensor integrals. The challenging step in this context is the numerically fast and stable reduction of the tensor integrals to scalar one-loop integrals. To ensure the correctness of our results we did two independent calculations of the virtual corrections using as far as possible different methods and also different tools. In one calculation the virtual corrections are essentially obtained following the procedure described in Ref. [11], where $t \bar{t}+\mathrm{H}$ production at hadron colliders was considered. Feynman diagrams and amplitudes have been generated with the FeynArts package [12[13] and further processed with in-house Mathematica routines, which automatically create an output in Fortran. The IR (soft and collinear) singularities are analytically separated from the finite remainder as described in Refs. [11|14]. The tensor integrals appearing in the pentagon diagrams are directly reduced to box integrals following Ref. [15]. This method does not introduce inverse Gram determinants in this step, thereby avoiding notorious numerical instabilities in regions where these determinants become small. Box and lower-point integrals are reduced à la Passarino-Veltman [16] to scalar integrals, which are either calculated analytically or using the results of Refs. [17/18 19]. Sufficient numerical stability is already achieved in this way. Nevertheless the integral evaluation is currently further refined by employing the more sophisticated methods described 
in Ref. [20] in order to numerically stabilize the tensor integrals in exceptional phase-space regions.

In the second calculation the evaluation of loop diagrams starts with the generation of diagrams and amplitudes via QGRAF [21], which are then further manipulated with Form [22] and automatically translated into $\mathrm{C}++$ code. The reduction of the 5-point tensor integrals to scalar integrals is performed with an extension of the method described in Ref. [23]. In this procedure also inverse Gram determinants of four four-momenta are avoided. The lower-point tensor integrals are reduced using an independent implementation of the Passarino-Veltman procedure. The IR-finite scalar integrals are evaluated using the FF package [2425].

\subsection{Real corrections}

The matrix elements for the real corrections are given by $0 \rightarrow \mathrm{t} \overline{\mathrm{t}} g g g g, 0 \rightarrow \mathrm{t} \overline{\mathrm{t}} q \bar{q} g g, 0 \rightarrow \mathrm{t} \overline{\mathrm{t}} q \bar{q} q^{\prime} \bar{q}^{\prime}$ and $0 \rightarrow \overline{\mathrm{t}} q \bar{q} q \bar{q}$. The various partonic processes are obtained from these matrix elements by all possible crossings of light particles into the initial state.

The evaluation of the real-emission amplitudes is again performed in two independent ways. Both evaluations employ the dipole subtraction formalism [26 27]28] for the extraction of IR singularities and for their combination with the virtual corrections.

One calculation of the real corrections results from a fully automated calculation based on helicity amplitudes, as described in Ref. [29]. Individual helicity amplitudes are computed with the help of BerendsGiele recurrence relations [9]. The evaluation of color factors and the generation of subtraction terms is automated. For the channel $g g \rightarrow \bar{t} \bar{t} g g$ a dedicated softinsertion routine [30] is used for the generation of the phase space. The second calculation uses for the LO $2 \rightarrow 3$ processes and the $g g \rightarrow \bar{t} \bar{g} g$ process optimized code obtained from a Feynman diagrammatic approach. As in the calculation described before standard techniques like color decomposition and the use of helicity amplitudes are employed. For the $2 \rightarrow 4$ processes including light quarks, Madgraph [10] has been used. The subtraction terms according to Ref. [28] are obtained in a semi-automatized manner based on an in-house library written in $\mathrm{C}++$.

\section{Results}

In the following we consistently use the CTEQ6 [31 32] set of parton distribution functions (PDFs). In detail, we take CTEQ6L1 PDFs with a 1-loop running $\alpha_{s}$ in LO and CTEQ6M PDFs with a 2-loop running $\alpha_{\mathrm{s}}$ in NLO. The number of active flavours is $N_{\mathrm{F}}=5$, and the respective QCD parameters are $\Lambda_{5}^{\mathrm{LO}}=165 \mathrm{MeV}$ and $\Lambda_{5}^{\overline{\mathrm{MS}}}=226 \mathrm{MeV}$. Note that the top-quark loop in the gluon self-energy is subtracted at zero momentum. In this scheme the running of $\alpha_{\mathrm{s}}$ is generated solely by the contributions of the light quark and gluon loops. The top-quark mass is renormalized in the on-shell scheme, as numerical value we take $m_{\mathrm{t}}=174 \mathrm{GeV}$.

We apply the jet algorithm of Ref. [33] with $R=$ 1 for the definition of the tagged hard jet. Unless stated otherwise we require a transverse momentum of $p_{\mathrm{T}, \mathrm{jet}}>p_{\mathrm{T}}^{\text {cut }}=20 \mathrm{GeV}$ for the hardest jet. The outgoing (anti-)top-quarks are neither affected by the jet algorithm nor by the phase-space cut. Note that the LO prediction and the virtual corrections are not influenced by the jet algorithm, but the real corrections are.

In Fig. 1 the scale dependence of the NLO cross sections is shown. For comparison, the LO results are included as well. Varying the scale in the usual range that is a factor 2 up and down around a central scale we observe a reduction of the scale dependence of a factor 3 at Tevatron and about a factor 6 at the LHC. From the absolute values of the cross sections one concludes that indeed a significant contribution to the inclusive top-quark pair cross section comes from $\mathrm{t} \overline{\mathrm{t}}+1$-jet. For reasonable scale choices one can also observe that the NLO corrections are of moderate size. In particular around $\mu=m_{\mathrm{t}}$ the corrections are small. The interference of $C$-odd contributions of the amplitude with $C$-even parts, where $C$ denotes the charge conjugation, produces a forwardbackward charge asymmetry at the Tevatron. For the inclusive top-quark pair sample, where this asymmetry is an NLO effect, this has been studied in Refs. [35 36 37]. In the case of $t \bar{t}+1$-jet this asymmetry appears already in LO. In LO the asymmetry is defined by

$A_{\mathrm{FB}, \mathrm{LO}}^{\mathrm{t}}=\frac{\sigma_{\mathrm{LO}}^{-}}{\sigma_{\mathrm{LO}}^{+}}, \quad \sigma_{\mathrm{LO}}^{ \pm}=\sigma_{\mathrm{LO}}\left(y_{\mathrm{t}}>0\right) \pm \sigma_{\mathrm{LO}}\left(y_{\mathrm{t}}<0\right),(1)$

where $y_{\mathrm{t}}$ denotes the rapidity of the top-quark. Cross- 

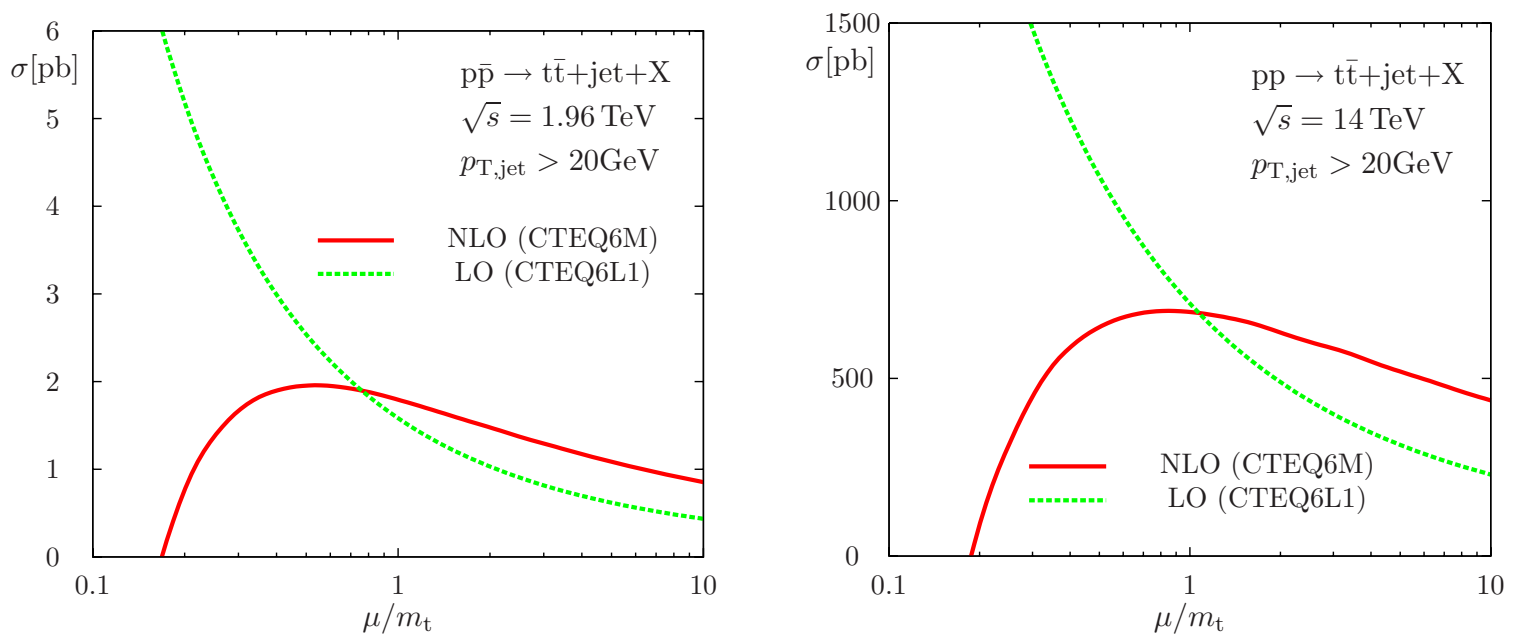

Figure 1. Scale dependence of the LO and NLO cross sections for $\mathrm{t} \overline{\mathrm{t}}+1$-jet production at the Tevatron (left) and the LHC (right) as taken from Ref. [34], with the renormalization scale $\left(\mu_{r}\right)$ and the factorization scale $\left(\mu_{f}\right)$ set to $\mu_{\text {. }}$

section contributions $\sigma\left(y_{\mathrm{t}} \gtrless 0\right)$ correspond to topquarks in the forward or backward hemispheres, respectively, where incoming protons fly into the forward direction by definition. Denoting the corresponding NLO contributions to the cross sections by $\delta \sigma_{\mathrm{NLO}}^{ \pm}$, we define the asymmetry at NLO by

$A_{\mathrm{FB}, \mathrm{NLO}}^{\mathrm{t}}=\frac{\sigma_{\mathrm{LO}}^{-}}{\sigma_{\mathrm{LO}}^{+}}\left(1+\frac{\delta \sigma_{\mathrm{NLO}}^{-}}{\sigma_{\mathrm{LO}}^{-}}-\frac{\delta \sigma_{\mathrm{NLO}}^{+}}{\sigma_{\mathrm{LO}}^{+}}\right)$,

i.e. via a consistent expansion in $\alpha_{\mathrm{s}}$. Note, however, that the LO cross sections in Eq. (2) are evaluated in the NLO setup (PDFs, $\alpha_{s}$ ). The results for the asymmetry for different scale choices are shown in Fig. 2. At LO we find an asymmetry of about $-8 \%$. The scale dependence is rather small. This is a consequence of the fact that $\alpha_{S}$ cancels exactly between the numerator and the denominator. In addition the residual factorization scale dependence also cancels to a large extent in the ratio. At NLO we find a large correction compared to the $\mathrm{LO}$ result. The asymmetry is almost washed out at NLO. The scale dependence is increased in NLO which seems natural given the small dependence in LO. To investigate the origin of the large NLO corrections to the asymmetry we studied the dependence on $p_{\mathrm{T}}^{\text {cut }}$, the minimal $p_{\mathrm{T}}$ used to resolve the additional jet. The results are shown in Tab. 1 A strong dependence of the cross section on $p_{\mathrm{T}}^{\text {cut }}$ is observed. For all $p_{\mathrm{T}}^{\text {cut }}$ values we find that the NLO corrections to the cross section are of moderate

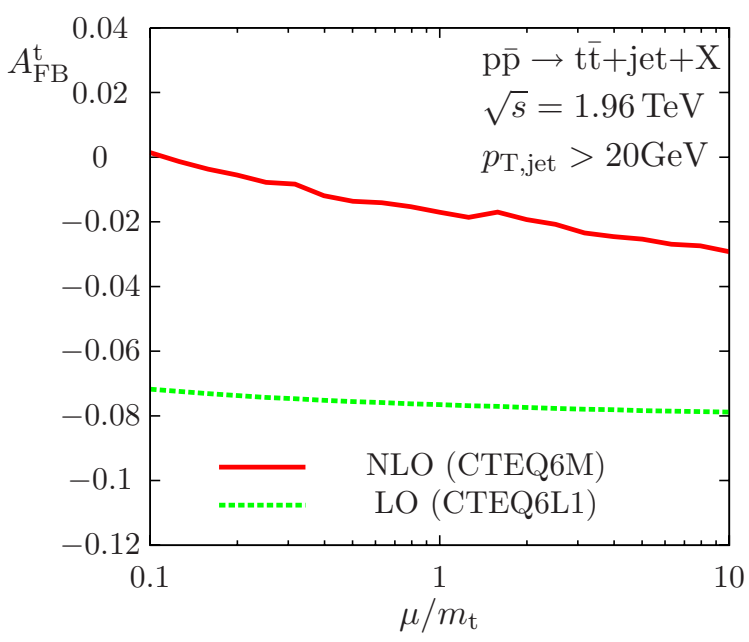

Figure 2. Scale dependence of the LO and NLO forward-backward charge asymmetry of the topquark in $\mathrm{p} \overline{\mathrm{p}} \rightarrow \mathrm{t} \overline{\mathrm{t}}+\mathrm{jet}+X$ at the Tevatron as taken from Ref. [34] with $\mu=\mu_{f}=\mu_{r}$. 
Table 1

Cross section and forward-backward charge asymmetry at the Tevatron for different values of $p_{\mathrm{T}}^{\text {cut }}$ used to define the minimal transverse momentum $p_{\mathrm{T}}$ of the additional jet $\left(\mu=\mu_{f}=\mu_{r}=m_{t}\right)$. The upper and lower indices are the shifts towards $\mu=m_{t} / 2$ and $\mu=2 m_{t}$.

\begin{tabular}{c|c|c|c|c}
\hline & \multicolumn{2}{|c|}{ cross section [pb] } & \multicolumn{2}{c}{ charge asymmetry [\%] } \\
$p_{\mathrm{T}}^{\text {cut }}[\mathrm{GeV}]$ & LO & NLO & LO & NLO \\
\hline 20 & $1.583(2)_{-0.55}^{+0.96}$ & $1.791(1)_{-0.31}^{+0.16}$ & $-7.69(4)_{-0.085}^{+0.10}$ & $-1.77(5)_{-0.30}^{+0.58}$ \\
30 & $0.984(1)_{-0.34}^{+0.60}$ & $1.1194(8)_{-0.20}^{+0.11}$ & $-8.29(5)_{-0.085}^{+0.12}$ & $-2.27(4)_{-0.51}^{+0.31}$ \\
40 & $0.6632(8)_{-0.23}^{+0.41}$ & $0.7504(5)_{-0.14}^{+0.072}$ & $-8.72(5)_{-0.10}^{+0.13}$ & $-2.73(4)_{-0.49}^{+0.35}$ \\
50 & $0.4670(6)_{-0.17}^{+0.29}$ & $0.5244(4)_{-0.096}^{+0.049}$ & $-8.96(5)_{-0.11}^{+0.14}$ & $-3.05(4)_{-0.39}^{+0.49}$ \\
\hline
\end{tabular}

size. While the cross section evidently has a large sensitivity on $p_{\mathrm{T}}^{\text {cut }}$, the dependence of the asymmetry on $p_{\mathrm{T}}^{\text {cut }}$ is slightly less pronounced. On the other hand we find, independent of the chosen $p_{\mathrm{T}}^{\text {cut }}$, a significant difference between LO and NLO for the asymmetry indicating that the definition of the asymmetry Eq. (2) is not stable with respect to higher-order corrections independent of the $p_{\mathrm{T}}^{\text {cut }}$ value. In Fig. 3 the rapidity distribution for the top-quark is shown. One can easily observe the asymmetry of the LO distribution leading to the aforementioned $-8 \%$ for the integrated asymmetry. The NLO corrections are different in shape compared to LO. They are larger in the forward direction than in the backward direction. The result is that the asymmetry is washed out by the NLO correction. In the lower plot of Fig. 3 the $K$-factor and the scale dependence is shown. The corrections are between +20 and $+40 \%$. The large scale dependence of the LO result is again significantly improved when the NLO corrections are taken into account. In Fig. 4 the $p_{\mathrm{T}}$-distribution of the top-quark is shown-now for the LHC. For not too large $p_{\mathrm{T}}$ the corrections are of moderate size. Starting with $+30 \%$ for small $p_{\mathrm{T}}$ and reaching $-50 \%$ at $p_{\mathrm{T}} \approx 450 \mathrm{GeV}$, indicating that the $K$-factor is strongly phase-space dependent. Up to a $p_{\mathrm{T}}$ of about $450 \mathrm{GeVwe}$ also find an important improvement of the scale uncertainty.

Conclusions: Predictions for $\overline{\mathrm{t}}+$ jet production at hadron colliders have been reviewed at NLO QCD. For the cross section the NLO corrections drastically reduce the scale dependence of the LO predictions, which is of the order of $100 \%$. The charge asymmetry of the top-quarks, which is measured at the Tevatron, is significantly decreased at NLO and is almost washed out by the residual scale dependence. In

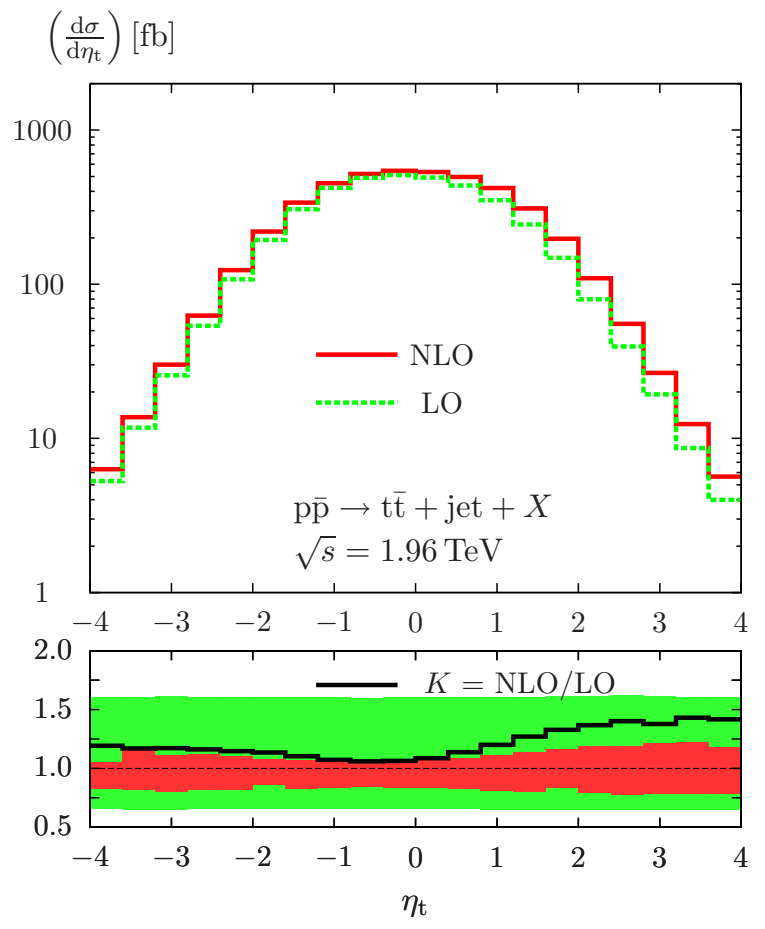

Figure 3. Rapidity distribution of the top-quark. In the upper plot the thick (thin) line shows the NLO (LO) result. In the lower plot the thick line shows the $K$-factor, the two bands show the scale dependence in LO (outer band) and NLO (inner band) when the scale $\left(\mu=\mu_{r}=\mu_{f}\right)$ is varied by factor 2 up and down around the central value $\mu=m_{\mathrm{t}}$. 


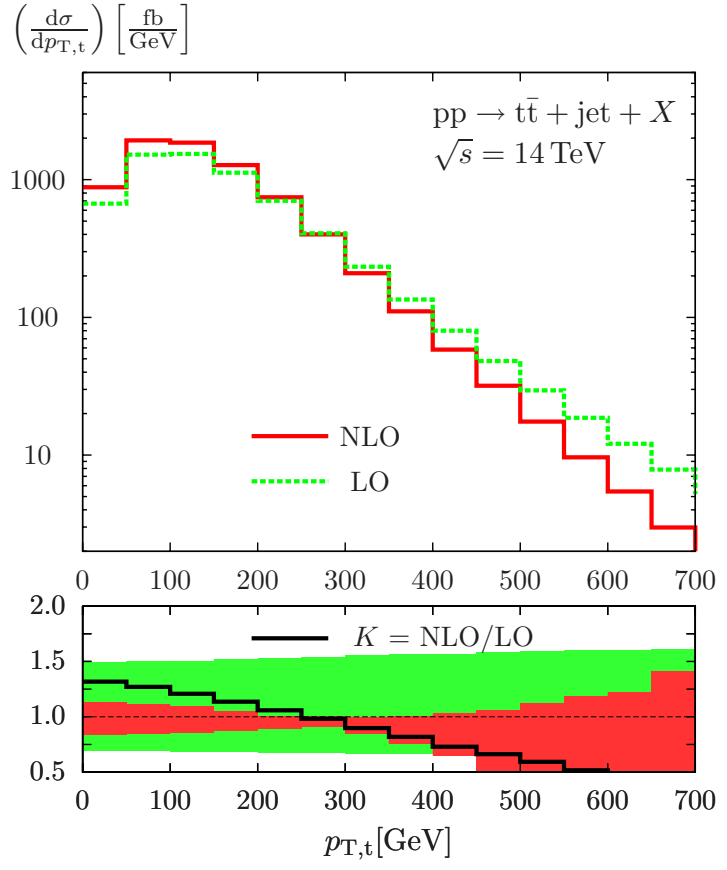

Figure 4. Same as Fig. 3 for the $p_{\mathrm{T}}$-distribution of the top-quark at LHC.

addition we have studied the $p_{\mathrm{T}}^{\text {cut }}$-dependence of the asymmetry in NLO. Further refinements of the precise definition of the charge asymmetry are required to stabilize the predictions with respect to higherorder corrections. First results for differential distributions have been presented. The corrections are well under control over a large phase-space region and the scale uncertainty is again improved compared to the LO results.

\section{REFERENCES}

1. W. Bernreuther, J. Phys. G35 (2008) 083001.

2. T. Han, (2008), arXiv 0804.3178.

3. S. Moch and P. Uwer, (2008), arXiv 0804.1476.

4. M. Cacciari et al., (2008), arXiv 0804.2800.

5. N. Kidonakis and R. Vogt, (2008), arXiv 0805.3844.

6. M. Czakon, A. Mitov and S. Moch, Phys. Lett. B651 (2007) 147, arXiv:0707.4139

7. M. Czakon, (2008), arXiv:0803.1400

8. A. Alves et al., Phys. Rev. D69 (2004) 075005.
9. F.A. Berends and W.T. Giele, Nucl. Phys. B306 (1988) 759.

10. T. Stelzer and W.F. Long, Comput. Phys. Commun. 81 (1994) 357.

11. W. Beenakker et al., Nucl. Phys. B653 (2003) 151.

12. J. Küblbeck, M. Böhm and A. Denner, Comput. Phys. Commun. 60 (1990) 165.

13. T. Hahn, Comput. Phys. Commun. 140 (2001) 418.

14. S. Dittmaier, Nucl. Phys. B675 (2003) 447.

15. A. Denner and S. Dittmaier, Nucl. Phys. B658 (2003) 175.

16. G. Passarino and M.J.G. Veltman, Nucl. Phys. B160 (1979) 151.

17. G. 't Hooft and M.J.G. Veltman, Nucl. Phys. B153 (1979) 365.

18. W. Beenakker and A. Denner, Nucl. Phys. B338 (1990) 349.

19. A. Denner, U. Nierste and R. Scharf, Nucl. Phys. B367 (1991) 637.

20. A. Denner and S. Dittmaier, Nucl. Phys. B734 (2006) 62.

21. P. Nogueira, J. Comput. Phys. 105 (1993) 279.

22. J.A.M. Vermaseren, (2000), math-ph/0010025.

23. W.T. Giele and E.W.N. Glover, JHEP 04 (2004) 029.

24. G.J. van Oldenborgh and J.A.M. Vermaseren, Z. Phys. C46 (1990) 425.

25. G.J. van Oldenborgh, Comput. Phys. Commun. 66 (1991) 1.

26. S. Catani and M.H. Seymour, Nucl. Phys. B485 (1997) 291.

27. L. Phaf and S. Weinzierl, JHEP 04 (2001) 006.

28. S. Catani et al., Nucl. Phys. B627 (2002) 189.

29. S. Weinzierl, Eur. Phys. J. C45 (2006) 745.

30. S. Weinzierl and D.A. Kosower, Phys. Rev. D60 (1999) 054028.

31. J. Pumplin et al., JHEP 07 (2002) 012.

32. D. Stump et al., JHEP 10 (2003) 046.

33. S.D. Ellis and D.E. Soper, Phys. Rev. D48 (1993) 3160.

34. S. Dittmaier, P. Uwer and S. Weinzierl, Phys. Rev. Lett. 98 (2007) 262002.

35. F. Halzen, P. Hoyer and C.S. Kim, Phys. Lett. B195 (1987) 74.

36. J.H. Kühn and G. Rodrigo, Phys. Rev. D59 (1999) 054017, Phys. Rev. Lett. 81 (1998) 49. 
37. M.T. Bowen, S.D. Ellis and D. Rainwater, Phys. Rev. D73 (2006) 014008. 